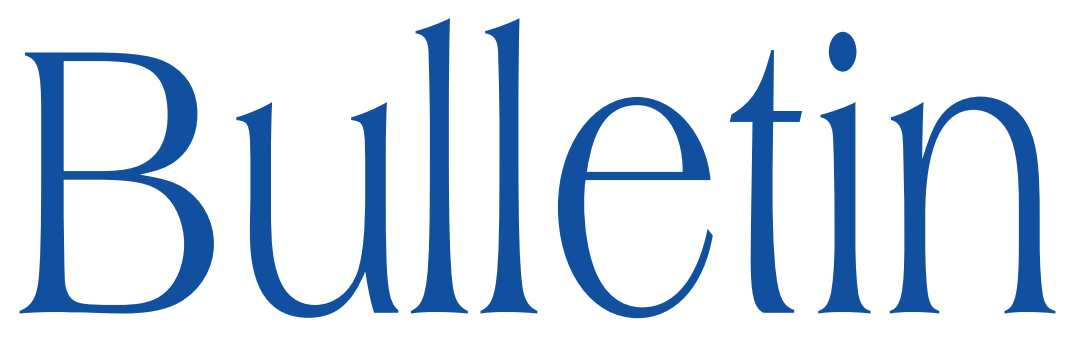

de la SOCIÉTÉ MATHÉMATIQUE DE FRANCE

\title{
ON BRODY AND ENTIRE CURVES
}

\author{
Jörg Winkelmann
}

\section{Tome 135}

Fascicule 1

2007

\section{SOCIÉTÉ MATHÉMATIQUE DE FRANCE}




\title{
ON BRODY AND ENTIRE CURVES
}

\author{
BY JÖRG WINKELMANN
}

\begin{abstract}
We discuss an example of an open subset of a torus which admits a dense entire curve, but no dense Brody curve.

RÉsumé (Sur les courbes de Brody et les courbes entières). - On présente un exemple de sous-ensemble de tore qui possède une courbe entière dense mais pas de courbe de Brody.
\end{abstract}

\section{Introduction}

1.1. Brody's theorem. - Let $Y$ be a complex manifold. It is called "taut" if the family of all holomorphic maps $f: \Delta \rightarrow Y$ is a normal family. Let us from now on assume that $Y$ is compact. Then being "taut" is easily seen to be equivalent with hyperbolicity in the sense of Kobayashi. The theorem of Brody (see [3]) states that this is furthermore equivalent with the property that every holomorphic map from $\mathbb{C}$ to $Y$ is constant. (Remark: This can be regarded as a special case of a heuristic philosophy known as "Bloch's principle", see [12].)

Now we may raise the question: What about holomorphic maps to a compact complex manifold fixing some given base points? Given a compact complex manifold $Y$ and a point $y \in Y$, let us consider the following two statements:

Texte reçu le 13 juin 2005, révisé le 4 octobre 2006

Jörg Winkelmann, Mathematisches Institut, Universität Bayreuth, Universitätsstraße 30, D-95447 Bayreuth, Germany, • E-mail : jwinkel@member.ams.org 2000 Mathematics Subject Classification. - 32A22, 32Q45.

Key words and phrases. - Brody lemma, entire curve, hyperbolicity, abelian variety. 
- Every holomorphic map $f: \mathbb{C} \rightarrow Y$ with $f(0)=y$ is constant.

- The family of all holomorphic maps $f: \Delta \rightarrow Y$ with $f(0)=y$ is a normal family.

Are they equivalent?

Using the notion of the infinitesimal Kobayashi-Royden pseudometric as introduced in [10] this can be reformulated into the following question: "If the infinitesimal Kobayashi-Royden pseudometric on a compact complex manifold $Y$ degenerates for some point $y \in Y$, does this imply that there exists a holomorphic map $f: \mathbb{C} \rightarrow Y$ with $y \in f(\mathbb{C})$ ?"

Thanks to Brody's theorem it is clear that there exists some non-constant holomorphic map $f: \mathbb{C} \rightarrow Y$ if the Kobayashi-Royden pseudometric is degenerate at some point $y$ of $Y$. But it is not clear that $f$ can be chosen in such a way that $y$ is in the image or at least in the closure of the image. Of course, at first it looks absurd that degeneracy of the Kobayashi-Royden pseudometric at one point $y$ should only imply the existence of a non-constant holomorphic map to some part of $Y$ far away of $y$ and should not imply the existence of a non-constant map $f: \mathbb{C} \rightarrow Y$ whose image comes close to $y$.

Thus one is led to ask:

Question 1. - Let $X$ be a compact complex manifold, $x \in X$. Assume that the infinitesimal Kobayashi-Royden pseudometric is degenerate on $T_{x} X$. Does this imply that there exists a non-constant holomorphic map $f: \mathbb{C} \rightarrow X$ with $f(0)=x$ ?

Since Brody parametrization always yields a holomorphic map $f: \mathbb{C} \rightarrow X$ whose derivative is bounded, one might be inclined to even ask:

Question 2. - Let $X$ be a compact complex manifold, $x \in X$. Assume that the infinitesimal Kobayashi-Royden pseudometric is degenerate on $T_{x} X$. Does this imply that there exists a non-constant holomorphic map $f: \mathbb{C} \rightarrow X$ with $f(0)=x$ such that the derivative $f^{\prime}: \mathbb{C} \rightarrow T X$ is bounded (with respect to the euclidean metric on $\mathbb{C}$ and some hermitian metric on $X)$ ?

In this article we give examples which show that the answer to the second question is negative. The answer to the first question remains open.

TOME $135-2007-\mathrm{N}^{\mathrm{O}} 1$ 
1.2. Reparametrization. - The key idea for proving Brody's theorem is the following: Let $f_{n}: \Delta \rightarrow Y$ be a non-normal family. Then we look for an increasing sequence of disk $\Delta_{r_{n}}$ which exhausts $\mathbb{C}$ (i.e. $\lim r_{n}=+\infty$ ) and a sequence of holomorphic maps $\alpha_{n}: \Delta_{r_{n}} \rightarrow \Delta$ such that a subsequence of $f_{n} \circ \alpha_{n}$ converges (locally uniformly) to a non-constant holomorphic map from $\mathbb{C}$ to $Y$.

In his proof Brody used combinations of affine-linear maps with automorphisms of the disk for the $\alpha_{n}$.

Zalcman [12] investigated other reparametrizations where the $\alpha_{n}$ themselves are affine-linear maps, a concept which has the advantage that it can also be applied to harmonic maps.

1.3. Subvarieties of abelian varieties. - Let $A$ be a complex abelian variety (i.e. a compact complex torus which is simultaneously a projective algebraic variety) and $X$ a subvariety. Let $E$ denote the union of all translates of complex subtori of $A$ which are contained in $X$. It is known that this union is either all of $X$ or a proper algebraic subvariety (see [6]).

Since $A$ is a compact complex torus there is a flat hermitian metric on $A$ induced by the euclidean metric on $\mathbb{C}^{g}$ via $A \simeq \mathbb{C}^{g} / \Gamma$. A holomorphic map $f: \mathbb{C} \rightarrow A$ has bounded derivative with respect to this metric if and only if it is induced by an affine-linear map from $\mathbb{C}$ to $\mathbb{C}^{g}$.

From this, one can deduce that $f(\mathbb{C}) \subset E$ for every holomorphic map $f: \mathbb{C} \rightarrow X$ with bounded derivative. In fact, $f(\mathbb{C}) \subset E$ holds for very holomorphic map $f: \mathbb{C} \rightarrow X$; this is a consequence of the theorem of Bloch-Ochiai.

It is thus natural to conjecture:

ConjeCture. - The Kobayashi-pseudodistance on $X$ is a distance outside $E$.

For example, this statement is a consequence of the more general conjecture VIII.I.4 by S. Lang [9]. In the context of classification theory the above statement has also been conjectured by F. Campana [4, §9.3].

In the spirit of the analogue between diophantine geometry and entire holomorphic curves as pointed out by Vojta [11], the conjecture above is also supported by the famous result of Faltings [5] with which he solved the Mordell conjecture. This result states the following: If we assume that $A$ and $X$ are defined over a number field $K$, then with only finitely many exceptions every $K$-rational point of $X$ is contained in $E$. 
1.4. The first example. - We construct an example of the following type: There is an abelian surface $T$ with open subsets $\Omega_{2} \subset \Omega_{1} \subset T$ such that there exists an entire curve $f: \mathbb{C} \rightarrow \Omega_{1}$ for which the image is dense in $\Omega_{1}$, but for every non-constant Brody curve $f: \mathbb{C} \rightarrow T$ whose image is contained in $\Omega_{1}$ the closure of the image is a compact complex curve inside of $\Omega_{2}$ (and $\Omega_{2}$ is not dense in $\Omega_{1}$ ).

1.5. The second example. - We show that by blowing up a suitably chosen curve on a suitably chosen three-dimensional abelian variety, one can obtain a compact complex manifold $X$ with a hypersurface $Z$ such that $Z$ contains the image of every non-constant Brody curve, although $X$ does admit an entire curve with dense image and the infinitesimal Kobayashi-Royden pseudometric vanishes identically on $X$.

1.6. Why two examples? - Although the second example suffices to show that the answer to the second question is negative (and thus to show that the behaviour of Brody curves is fundamentally different from that of arbitrary entire curves), we include a description of the first example (which was obtained earlier), because we feel that it is of independent interest. The methods for constructing the two examples are completely different, and the first example might also be interesting from the point of view of studying entire curves in compact complex tori.

For example, for every entire curve with values in a compact complex torus the Zariski closure of the image is a translated subtorus, but our example shows that the ordinary closure of the image can be far away from being a translated real subtorus.

\section{Some basic facts on Brody curves}

We recall some basic facts on Brody curves.

Let $X$ be a complex manifold endowed with some hermitian metric. Then an entire curve is a non-constant holomorphic map from $\mathbb{C}$ to $X$ and a Brody curve is a non-constant holomorphic map $f: \mathbb{C} \rightarrow X$ for which the derivative $f^{\prime}$ is bounded (with respect to the euclidean metric on $\mathbb{C}$ and the given hermitian metric on $X)$.

- If $X$ is compact, the notion of a "Brody curve" is independent of the choice of the hermitian metric.

- If $\phi: X \rightarrow Y$ is a holomorphic map between compact complex manifolds and $f: \mathbb{C} \rightarrow X$ is a Brody curve, then $\phi \circ f: \mathbb{C} \rightarrow Y$ is a Brody curve, too. (But not necessarily conversely.)

TOME $135-2007-\mathrm{N}^{\circ} 1$ 
- If $X=\mathbb{C}^{g} / \Gamma$ is a compact complex torus (e.g. an abelian variety), then an entire curve $f: \mathbb{C} \rightarrow X$ is a Brody curve if and only if it lifts to an affine linear map $\tilde{f}: \mathbb{C} \rightarrow \mathbb{C}^{g}$.

\section{The first example}

3.1. Statement of the first main result. - We construct an example of an open domain in a torus for which the Brody reparametrization necessarily fundamentally changes the image for certain entire curves.

THEOREM 1. - There exists a compact complex torus $T$, equipped with a flat hermitian metric $h$ and open subsets $\Omega_{2} \subset \Omega_{1} \subset T$ such that:

1) $\Omega_{2}$ is not dense in $\Omega_{1}$ and neither is $\Omega_{1}$ in $T$.

2) For every point $p \in \Omega_{1}$ and every $v \in T_{p} \Omega_{1}$ there is a non-constant holomorphic map $f: \mathbb{C} \rightarrow \Omega_{1}$ with $p=f(0), v=f^{\prime}(0)$ and $\bar{\Omega}_{1}=\overline{f(\mathbb{C})}$.

3) If $f: \mathbb{C} \rightarrow T$ is a non-constant holomorphic map with bounded derivative (with respect to the euclidean metric on $\mathbb{C}$ and $h$ on $T$ ) and $f(\mathbb{C}) \subset \bar{\Omega}_{1}$, then $f(\mathbb{C}) \subset \bar{\Omega}_{2}$. Moreover $f$ is affine-linear and $\overline{f(\mathbb{C})}$ is a closed analytic subset of $T$.

We remark that this implies in particular that the infinitesimal KobayashiRoyden pseudometric vanishes identically on $\Omega_{1}$.

Furthermore, it provides examples of holomorphic maps from $\mathbb{C}$ into a compact complex torus with a rather "bad" image: The closure of the image with respect to the euclidean topology is $\bar{\Omega}_{2}$ and thus a set with non-empty interior such that the complement has also non-empty interior. This is in strong contrast to the Zariski-analytic closure: By the theorem of Green-Bloch-Ochiai for every holomorphic map $f$ from $\mathbb{C}$ to a compact complex torus $T$ the closure of the image $f(\mathbb{C})$ with respect to the analytic Zariski topology (i.e. the smallest closed analytic subset of $T$ containing $f(\mathbb{C})$ ) is always a translated subtorus of $T$.

We will now describe our example.

We precede the construction with some elementary observations about tori: Let $T=\mathbb{C}^{n} / \Lambda$ be a torus, equipped with the flat euclidean metric and the corresponding distance function $d_{T}(.,$.$) . Let$

$$
\rho=\frac{1}{2} \min _{\gamma \in \Lambda \backslash\{0\}}\|\gamma\| .
$$


This is the injectivity radius, in other words $\rho$ is the largest real number such that the natural projection $\pi: \mathbb{C}^{n} \rightarrow T$ induces for all $\left.\epsilon<\rho 2 \mathrm{pt}\right][2 \mathrm{pt}]$ a homeomorphism between the ball

$$
B_{\epsilon}\left(\mathbb{C}^{n} ; 0\right)=\left\{v \in \mathbb{C}^{n}:\|v\|<\epsilon\right\}
$$

and

$$
B_{\epsilon}(T ; e)=\left\{x \in T: d_{T}(x, e)<\epsilon\right\} .
$$

Evidently, the injectivity radius $\rho$ is a lower bound for the diameter

$$
\rho \leq \operatorname{diam}=\max _{x, y \in T} d_{T}(x, y) .
$$

If we pass from $T$ to a subtorus $S \subset T$, the injectivity radius can only increase, while the diameter can only decrease. As a consequence we obtain:

LEMMA 1. - Let $T$ be a compact (real or complex) torus with injectivity radius $\rho$. Then for every real positive-dimensional subtorus $S \subset T$ the diameter

$$
\operatorname{diam}(S)=\max _{x, y \in S} d_{T}(x, y)
$$

is at least $\rho$. Furthermore, if $0<\epsilon \leq \rho$ and $x \in T$, then the ball $B_{\epsilon}(T ; x)$ contains no translate of any positive-dimensional real subtorus of $T$.

Before giving the details of the construction of our example, let us try to express its idea in a drawing (see Figure 1).

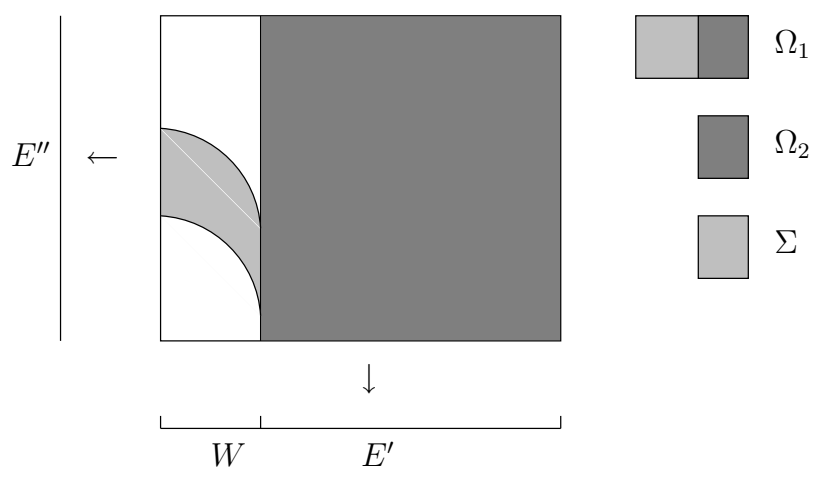

Figure 1.

Now let us start the precise construction of the example.

Let $E^{\prime}=\mathbb{C} / \Gamma^{\prime}$ and $E^{\prime \prime}=\mathbb{C} / \Gamma^{\prime \prime}$ be elliptic curves and $T=E^{\prime} \times E^{\prime \prime}$.

Let $\pi^{\prime}: \mathbb{C} \rightarrow E^{\prime}, \pi^{\prime \prime}: \mathbb{C} \rightarrow E^{\prime \prime}$ and $\pi=\left(\pi^{\prime}, \pi^{\prime \prime}\right): \mathbb{C}^{2} \rightarrow T$ denote the natural projections.

TOME $135-2007-\mathrm{N}^{\mathrm{O}} 1$ 
We assume that $E^{\prime}$ is not isogenous to $E^{\prime \prime}$. (For example, we might choose $E^{\prime}=\mathbb{C} / \mathbb{Z}[i]$ and $E^{\prime \prime}=\mathbb{C} / \mathbb{Z}[\sqrt{2} i]$.) Then $E^{\prime} \times\{0\}$ and $\{0\} \times E^{\prime \prime}$ are the only non-trivial complex subtori of $T$.

Now $T=\mathbb{C}^{2} / \Gamma$ with $\Gamma=\Gamma^{\prime} \times \Gamma^{\prime \prime}$. The compact complex torus $T$ carries a hermitian metric $h$ induced by the euclidean metric on $\mathbb{C}^{2}$ (i.e. $h=\mathrm{d} z_{1} \otimes \mathrm{d} \bar{z}_{1}$ $\left.+\mathrm{d} z_{2} \otimes \mathrm{d} \bar{z}_{2}\right)$. The associated distance function is called $d$, the injectivity radius $\rho$ is defined as explained above.

We choose numbers $0<\rho^{\prime}<\rho^{\prime \prime}<\rho$ and define

$$
W=B_{\rho^{\prime}}\left(E^{\prime}, e\right) .
$$

Furthermore we choose $0<\delta<\frac{1}{2} \rho$ and a holomorphic map $\sigma: \mathbb{C} \rightarrow E^{\prime \prime}$ such that there exist complex numbers $t, t^{\prime} \in B_{\rho^{\prime}}(\mathbb{C}, 0)$ (i.e. $\left.|t|,\left|t^{\prime}\right|<\rho^{\prime}\right)$ and

$$
d_{E^{\prime \prime}}\left(\sigma(t), \sigma\left(t^{\prime}\right)\right)>2 \delta \text {. }
$$

(This is possible since $2 \delta$ is smaller than the injectivity radius $\rho$ of $T$ which in turn is a lower bound for the diameter of $\left.E^{\prime \prime}\right)$.

We denote by $s: \mathbb{C} \rightarrow \mathbb{C}$ a holomorphic function such that $\sigma=\pi^{\prime \prime} \circ s$.

Since $\pi^{\prime}: \mathbb{C} \rightarrow E^{\prime}$ restricts to an isomorphism between $B_{\rho}(\mathbb{C}, 0)$ and $B_{\rho}\left(E^{\prime}, e\right)$, the holomorphic maps $s$ and $\sigma$ induce maps from $B_{\rho}\left(E^{\prime}, e\right)$ to $\mathbb{C}$ (resp. $E^{\prime \prime}$ ). By abuse of notation these maps will also be denoted by $s$ (resp. $\sigma$ ).

Now define $\Omega_{2}=\left(E^{\prime} \backslash \bar{W}\right) \times E^{\prime \prime}$ and $\Omega_{1}=\Omega_{2} \cup \Sigma$ with

$$
\Sigma=\left\{(x, y): x \in \bar{W}, y \in E^{\prime \prime}, d_{E^{\prime \prime}}(y-\sigma(x))<\delta\right\} .
$$

Let us now fix some point $p \in \Omega_{1}$ and $v=\left(v_{1}, v_{2}\right) \in T_{p}(T)=\mathbb{C}^{2}$. We have to show that there exists a holomorphic map $f$ as stipulated in 2) of theorem 1.

Let $\left(p_{1}, p_{2}\right) \in \mathbb{C}^{2}$ be a point mapped on $p$ by $\pi: \mathbb{C}^{2} \rightarrow T$. If $p \in \Sigma$, we require $\left|p_{1}\right| \leq \rho^{\prime}$ and $\left|s\left(p_{1}\right)-p_{2}\right|<\delta$ and define $\delta^{\prime}=\delta-\left|s\left(p_{1}\right)-p_{2}\right|$. If $p \notin \Sigma$, we require $\left|p_{1}\right|>\rho^{\prime \prime}$ and define $\delta^{\prime}=\delta$.

As the next step, we will choose a pair of entire functions $(Q, H)$.

Claim 1. - There is a pair of entire functions $(Q, H)$ with the following properties:

1) $Q$ is a non-constant polynomial.

2) $(Q(0), H(0))=\left(p_{1}, p_{2}\right)$.

3) $\left(Q^{\prime}(0), H^{\prime}(0)\right)$ and $v$ are parallel.

4) If $p \in \Sigma$, we require furthermore that

$$
(Q(z), H(z)+y) \in \pi^{-1}(\Sigma)
$$

for all $z$ and $y$ with $|Q(z)| \leq \rho^{\prime}$ and $|y| \leq \frac{1}{2} \delta^{\prime}$. 
Proof of the claim. - Let us first discuss the case where $p \notin \Sigma$. Then it suffices to choose

$$
Q(z)=z^{2}+v_{1} z+p_{1} \text { and } H(z)=v_{2} z+p_{2} .
$$

If $p \in \Sigma$, we proceed as follows: First, for $r, t \in \mathbb{C}$ we define

$$
Q_{t}(z)=(z+t)^{2}+p_{1}-t^{2} \quad \text { and } \quad H_{r, t}(z)=p_{2}-s\left(p_{1}\right)+s\left(Q_{t}(z)\right)+r z .
$$

We will set $Q=Q_{t}$ and $H=H_{r, t}$ for appropriately chosen parameters $r, t$. Evidently $Q_{t}$ is a polynomial for any choice of $t$. Furthermore

$$
\left(Q_{t}(0), H_{r, t}(0)\right)=\left(p_{1}, p_{2}\right)
$$

independent of the choice of $r, t$ :

$$
Q_{t}(0)=t^{2}+p_{1}-t^{2}=p_{1} \quad \text { and } \quad H_{r, t}(0)=p_{2}-s\left(p_{1}\right)+s\left(p_{1}\right)+0=p_{2} .
$$

Let $\Phi_{r, t}=\left(Q_{t}, H_{r, t}\right)$. We have

$$
\Phi_{r, t}^{\prime}(0)=\left(Q_{t}^{\prime}(0), s^{\prime}\left(Q_{t}(0)\right) Q_{t}^{\prime}(0)+r\right)=\left(2 t, 2 s^{\prime}\left(p_{1}\right) t+r\right) .
$$

Observe that

$$
(r, t) \longmapsto \frac{2 t}{2 s^{\prime}\left(p_{1}\right) t+r}
$$

defines a meromorphic function on $\mathbb{C}^{2}$ with a point of indeterminacy at $(0,0)$. This is true regardless of the value of $s^{\prime}\left(p_{1}\right)$.

Thus every neighborhood of $(0,0)$ contains a point $(r, t) \neq(0,0)$ such that $\Phi_{r, t}^{\prime}(0)$ is a non-zero multiple of $v$.

Next we note that $(t, z) \mapsto Q_{t}(z)$ defines a proper map from $\overline{B_{1}(\mathbb{C}, 0)} \times \mathbb{C}$ to $\mathbb{C}$. Therefore there is a constant $C>0$ such that $|z|<C$, whenever there exists a parameter $t$ such that $|t| \leq 1$ and $\left|Q_{t}(z)\right| \leq \rho$.

It is therefore possible to choose two numbers $r, t$ in such a way that

1) $\Phi_{r, t}^{\prime}(0)$ is a non-zero multiple of $v$,

2) $|t|<1$ and

3) $|2 r C|<\delta^{\prime}$.

Now assume that $z, y \in \mathbb{C}$ with $\left|Q_{t}(z)\right| \leq \rho^{\prime}$ and $|y|<\frac{1}{2} \delta^{\prime}$. By the definition of the constant $C$, this implies $|z|<C$. Let

$$
\left(w_{1}, w_{2}\right)=\Phi_{r, t}(z)+(0, y) .
$$

Then

$$
\begin{aligned}
\left|w_{2}-s\left(w_{1}\right)\right| & =\left|p_{2}-s\left(p_{1}\right)+r z+y\right| \\
& <\left|p_{2}-s\left(p_{1}\right)\right|+|r C|+\frac{1}{2} \delta^{\prime}<\left(\delta-\delta^{\prime}\right)+\frac{1}{2} \delta^{\prime}+\frac{1}{2} \delta^{\prime}=\delta .
\end{aligned}
$$

Now $\left|w_{2}-s\left(w_{1}\right)\right|<\delta$ in combination with $\left|w_{1}\right|=\left|Q_{t}(z)\right| \leq \rho^{\prime}$ implies $\pi\left(w_{1}, w_{2}\right) \in \Sigma$. Hence $\Phi_{r, t}(z)+(0, y) \in \pi^{-1}(\Sigma)$ under this assumption. Thus the claim is proved. 
Our next step is to construct a closed subset $A$ of $\mathbb{C}$ to which we will apply Arakelyan approximation.

Let $A_{0}$ be the union of $\overline{B_{\rho^{\prime \prime}}(0)}$ and $\overline{B_{\rho^{\prime}}(\gamma)}$ for all $\gamma \in \Gamma^{\prime}$. If $p \notin \Sigma$, then $p_{1} \notin A_{0}$. Hence in this case we can choose $\eta>0$ such that $\overline{B_{\eta}\left(p_{1}\right)}$ is disjoint to $A_{0}$ and define $A_{1}$ as the union of $A_{0}$ with this closed ball $\overline{B_{\eta}\left(p_{1}\right)}$. If $p \in \Sigma$, we simply take $A_{1}=A_{0}$.

Next we choose dense countable subsets $S_{1} \subset \operatorname{int}(\Sigma)$ (where $\operatorname{int}(\Sigma)$ denotes the interior of $\Sigma$ ) and $S_{2} \subset \Omega_{2}$. We observe that $\mathbb{C} \backslash A_{1}$ projects surjectively onto $E^{\prime} \backslash \bar{W}$ and that the fibers of this projection are infinite discrete subsets of $\mathbb{C}$. For this reason we can find sequences $a_{n}, b_{n}$ in $\mathbb{C}$ such that

$$
S_{2}=\left\{\pi\left(a_{n}, b_{n}\right): n \in \mathbb{N}\right\}
$$

and all the $a_{n}$ are distinct elements of $\mathbb{C} \backslash A_{1}$ with $\lim _{n \rightarrow \infty}\left|a_{n}\right|=+\infty$. It follows that

$$
\Theta=\left\{a_{n}: n \in \mathbb{N}\right\}
$$

is a discrete subset of $\mathbb{C}$ which has empty intersection with $A_{1}$. We define

$$
A_{2}=A_{1} \cup \Theta \text {. }
$$

We fix a bijection $\xi: \Gamma^{\prime} \backslash\{0\} \stackrel{\sim}{\rightarrow} S_{1}$ and an enumeration $n \mapsto \gamma_{n}$ of $\Gamma^{\prime} \backslash\{0\}$. Then we can choose sequences of complex numbers $c_{n}, d_{n}$ such that the following properties hold for all $n \in \mathbb{N}$

1) $\pi\left(c_{n}, d_{n}\right)=\xi\left(\gamma_{n}\right)$,

2) $\left|c_{n}-\gamma_{n}\right|<\rho^{\prime}$ and

3) $\left|d_{n}-s\left(c_{n}\right)\right|<\delta$.

We define $A=Q^{-1}\left(A_{2}\right)$. Observe that $A$ contains $Q^{-1}\left(S_{1}\right)$.

Claim 2. - Arakelyan approximation is applicable to $A$, i.e. $\{\infty\} \cup(\mathbb{C} \backslash A)$ is connected and locally connected.

Proof of the claim. - Observe that $\mathbb{C} \backslash A_{1}$ is an unbounded open connected subset and that $Q: \mathbb{C} \rightarrow \mathbb{C}$ is a ramified covering. Hence the connected components of $Q^{-1}\left(\mathbb{C} \backslash A_{1}\right)$ are also unbounded. Thus $\mathbb{P}_{1} \backslash Q^{-1}(A)$ is connected. Furthermore $Q$ is conjugated to $z \mapsto z^{2}$ near $\infty$, which implies that $\mathbb{P}_{1} \backslash Q^{-1}(A)$ is locally connected at $\infty$. Thus the claim is proved.

We will now define a continuous function $h$ on $A$, which is holomorphic in its interior, and which we will then approximate by an entire function, using Arakelyan's theorem.

- If $p \notin \Sigma$, we take $h(z)=H(z)$ on $Q^{-1}\left(\overline{B_{\eta}\left(p_{1}\right)}\right)$ and $h=s$ on $Q^{-1}\left(\overline{B_{\rho^{\prime \prime}}(0)}\right)$.

- If $p \in \Sigma$, we define $h$ on $Q^{-1}\left(\overline{B_{\rho^{\prime \prime}}(0)}\right)$ as $H(z)$. 
Next, for every $n \in \mathbb{N}$, we define $h(z)$ as

$$
h(z)=s\left(Q(z)-\gamma_{n}\right)+d_{n}-s\left(c_{n}\right)
$$

whenever $\left|Q(z)-\gamma_{n}\right| \leq \rho^{\prime}$.

Finally, we define $h$ on $Q^{-1}(\Theta)$ by stipulating that $h(z)=b_{n}$ whenever $Q(z)=a_{n}$ for a number $n \in \mathbb{N}$.

By the construction of $(Q, H)$ we know that $\pi(Q(0), h(0))=p$ and that $\left(Q^{\prime}(0), h^{\prime}(0)\right)$ is a multiple of $v$. The choice of $h$ implies moreover that $S_{1} \cup S_{2}$ is contained in the image of $z \mapsto \pi(Q(z), h(z))$.

Next we define a continuous positive function $\epsilon: A \rightarrow \mathbb{R}^{+}$as follows:

- $\epsilon \equiv 1$ on $Q^{-1}\left(\overline{B_{\eta}\left(p_{1}\right)}\right)$ if $p \notin \Sigma$.

- $\epsilon \equiv \frac{1}{2} \delta^{\prime}$ on $Q^{-1}\left(\overline{B_{\rho^{\prime \prime}}(0)}\right)$.

- $\epsilon(z)=\frac{1}{n}$ if $Q(z)=a_{n}$.

- $\epsilon(z)=\min \left\{\frac{1}{n}, \frac{1}{2}\left(\delta-\left|d_{n}-s\left(c_{n}\right)\right|\right)\right\}$ whenever $\left|Q(z)-\gamma_{n}\right| \leq \rho^{\prime}$.

Using proposition 1 , we deduce that there exists an entire function $F: \mathbb{C} \rightarrow \mathbb{C}$ such that

1) $|F(z)-h(z)|<\epsilon(z)$ for all $z \in A$,

2) $F(0)=h(0)$ and $F^{\prime}(0)=h^{\prime}(0)$.

By the second condition we obtain that $\pi(Q(0), F(0))=p$ and that $\left(Q^{\prime}(0), F^{\prime}(0)\right)$ is a multiple of $v$. The first condition ensures that $\pi(Q(z), F(z))$ belongs to $\Omega$ for all $z \in \mathbb{C}$. It also ensure that the image is dense: Indeed, let $w \in \Omega_{2}$. Then there is a sequence of points in $S_{2}$ converging to $w$. But $S_{2}=\left\{\pi\left(a_{n}, b_{n}\right): n \in \mathbb{N}\right\}$ and the construction of $F$ implies that for every $n \in \mathbb{N}$ there exists a number $z_{n} \in \mathbb{C}$ such that $Q\left(z_{n}\right)=a_{n}$ and $\left|F\left(z_{n}\right)-b_{n}\right|<\frac{1}{n}$. It follows that there is a subsequence $z_{n_{k}}$ such that $\lim _{k} \pi\left(Q\left(z_{n_{k}}\right), F\left(z_{n_{k}}\right)\right)=w$. If $w \in \Sigma$, we argue similarily, with $S_{1}$ in the role of $S_{2}$. Thus the whole set $\Omega_{1}$ is in the closure of the image of the map $z \mapsto \pi(Q(z), F(z))$ from $\mathbb{C}$ to $T$.

Finally, let $\mu$ be a complex number such that $\mu\left(Q^{\prime}(0), F^{\prime}(0)\right)=v$ and define

$$
f(z)=\pi(Q(\mu z), F(\mu z)) .
$$

Then $f: \mathbb{C} \rightarrow \Omega_{1}$ is a holomorphic map with the desired properties.

We still have to show assertion 3 ) of the theorems. Recall that $T \simeq E^{\prime} \times$ $E^{\prime \prime}$ where $E^{\prime}$ and $E^{\prime \prime}$ are non-isogenous elliptic curves. Recall furthermore that $T, E^{\prime}, E^{\prime \prime}$ and $\{e\}$ are the only subtori of $T$ (this is a consequence of $E^{\prime}$ and $E^{\prime \prime}$ being non-isogenous). If $f: \mathbb{C} \rightarrow T$ is a holomorphic map with bounded derivative, Liouville's theorem implies that $f^{\prime}: \mathbb{C} \rightarrow \mathbb{C}^{2}$ is constant. Therefore $f$ is induced by an affine-linear map $\tilde{f}: \mathbb{C} \rightarrow \mathbb{C}^{2}$ and thus $f(\mathbb{C})$ is the orbit of a 1-dimensional complex Lie subgroup $H$ of $T$. Hence the closure $\bar{\Omega}_{1}$ must contain an orbit of the closure $\bar{H}$ of $H$ in $T$ if $f(\mathbb{C}) \subset \Omega_{1}$. We choose a point $w \in W$ and consider the intersection of this $\bar{H}$-orbit with $F=$ 
$\left(\pi^{\prime}\right)^{-1}(w) \simeq E^{\prime \prime}$. By construction $\Omega_{1} \cap F$ is an open disc embedded into the elliptic curve $E^{\prime \prime}$. Thus $F \cap \bar{\Omega}_{1}$ does not contain any orbit of any positivedimensional subtorus of $E^{\prime \prime}$. This leaves two possibilities: First, $f(\mathbb{C}) \cap F$ may be empty. Since every non-surjective holomorphic map from $\mathbb{C}$ to an elliptic curve is constant, it follows that $f(\mathbb{C})$ is a fiber of $\pi^{\prime}: T \rightarrow E^{\prime}$. As a consequence, $f(\mathbb{C}) \subset \Omega_{2}$ if $f(\mathbb{C}) \subset \Omega_{1}$. Second possibility: $f(\mathbb{C}) \cap F$ is nonempty, but finite. Then the Lie group homomorphism from $H$ to $E^{\prime}$ induced by the projection map $\pi^{\prime}$ has finite kernel. It follows that $H=\bar{H}=E^{\prime}$. However, by construction $\Omega_{1}$ does not contain any $E^{\prime}$-orbit. So the second possibility does not occur.

This completes the proof of theorems 1 and 2 (modulo the approximation result which we prove in the next section).

\section{Arakelyan approximation with interpolation}

We will need the following slight improvement of Arakelyan's theorem.

Proposition 1. - Let $A$ be a closed subset in $\mathbb{C}$ for which $\mathbb{P}_{1} \backslash A$ is connected and locally connected at $\infty$. Let $q$ be a point in the interior of $A$ and let $f: A \rightarrow \mathbb{C}$ be a continuous function which is holomorphic in the interior of $A$. Furthermore let $\epsilon: A \rightarrow \mathbb{R}^{+}$be a continuous function. Then there exists an entire function $F$ such that

$$
F(q)=f(q), \quad F^{\prime}(q)=f^{\prime}(q) \quad \text { and } \quad|F(z)-f(z)|<\epsilon(z)
$$

for all $z \in A$.

Proof. - By the classical theorem of Arakelyan (see [1]) we know that there is an entire function $F$ with $|F(z)-f(z)|<\epsilon(z)$ for all $z \in A$.

We have to show that we can choose $F$ in such a way as to obtain the additional conditions $F(q)=f(q)$ and $F^{\prime}(q)=f^{\prime}(q)$.

There is no loss in generality in assuming $f(q)=f^{\prime}(q)=0$ and $B_{1}(q) \subset A$.

First we show that we can achieve $F(q)=f(q)$. Let $F_{1}$ be an entire function with $\left|F_{1}(z)-f(z)\right|<\frac{1}{5} \epsilon(z)$ for all $z \in A$. If $F_{1}(q)=0$, we are done. If not, we define $g_{1}=F_{1}-f$ and choose an entire function $G_{1}$ such that

$$
\left|G_{1}(z)-g_{1}(z)\right|<\min \left\{\frac{1}{2}\left|g_{1}(q)\right|, \frac{1}{5} \epsilon(z)\right\}, \quad \forall z \in A .
$$

Then we define

$$
F_{2}=F_{1}-\frac{F_{1}(q)}{G_{1}(q)} G_{1} .
$$

By construction we have $\left|F_{1}(q) / G_{1}(q)\right|<2,\left|G_{1}(z)\right|<\frac{2}{5} \epsilon(z)$ and therefore $\left|F_{2}(z)-f(z)\right|<\epsilon(z)$ for all $z \in A$. Thus it is possible to find such an entire function with the additional property $F(q)=0=f(q)$. 
Next we discuss the condition on $F^{\prime}(q)$. By the preceding arguments there is an entire function $F_{2}$ with $F_{2}(q)=f(q)$ and $\left|F_{2}(z)-f(z)\right|<\frac{1}{5} \epsilon(z)$. Assume $F_{2}^{\prime}(q) \neq 0$. Then we define $g_{2}=F_{2}-f$ and choose an entire function $G_{2}$ with $G_{2}(q)=g_{2}(q)=0$ and

$$
\left|G_{2}(z)-g_{2}(z)\right|<\min \left\{\frac{1}{2}\left|g_{2}^{\prime}(q)\right|, \frac{1}{5} \epsilon(z)\right\}, \quad \forall z \in A .
$$

Since $B_{1}(q) \subset A$ and therefore $\left|G_{2}(z)-g_{2}(z)\right|<\frac{1}{2} g_{2}^{\prime}(q)$ for all $z \in B_{1}(q)$, the lemma of Schwarz implies that $\left|G_{2}^{\prime}(q)\right|>\frac{1}{2}\left|g_{2}^{\prime}(q)\right|$. Using this fact, one verifies easily that

$$
F_{3}(z)=F_{2}(z)-\frac{F_{2}^{\prime}(q)}{G_{2}^{\prime}(q)} G_{2}(z)
$$

is an entire function with the desired properties.

\section{The second main result}

We prove the following theorem:

TheOREM 2. - There exists a projective manifold $X$ with a hypersurface $Z$ such that for every point $x \in X$ there exists an entire curve $f: \mathbb{C} \rightarrow X$ with $\overline{f(\mathbb{C})}=X$ and $x \in f(\mathbb{C})$, but $Z$ contains the image of every Brody curve.

5.1. Proof of the main theorem. - The main theorem is a consequence of the more specific theorem below.

Theorem 3. - There exists an abelian threefold $A$ with a smooth curve $C$ such that the smooth projective variety $\widehat{A}$ obtained by blowing up $A$ along $C$ has the following properties:

1) For every point $p \in \widehat{A}$ there exists a non-constant entire curve $\gamma: \mathbb{C} \rightarrow \widehat{A}$ with $p \in \gamma(\mathbb{C})$ and $\overline{\gamma(\mathbb{C})}=\widehat{A}$.

2) For a given point $p \in \widehat{A}$ there exists a non-constant Brody curve $\gamma: \mathbb{C} \rightarrow \widehat{A}$ with $p \in \gamma(\mathbb{C})$ if and only if $p$ is contained in the exceptional divisor $E=\pi^{-1}(C)$ of the blow-up $\pi: \widehat{A} \rightarrow A$.

The key idea is that the hermitian metric will explode in some directions due to the blow up and that this will create an obstruction against lifting Brody curves. As a consequence there will be no Brody curves outside the exceptional divisor. To realize this idea it is necessary to ensure that the center of the blowup intersects the closure of the image of each Brody curve. To achieve this it will be necessary to blow up a center of positive dimension. Furthermore, since the center of a blow-up has real codimension at least four, it is necessary to choose the abelian variety in such a way that for every Brody curve the closure is at least real 4-dimensional. 
Proof. - 1) For every $x \in A$ and $v \in T_{x} A$ there is an affine-linear curve $\gamma: \mathbb{C} \rightarrow A$ with $\gamma(0)=x$ and $\gamma^{\prime}(0)=v$. Recall that $\pi: \widehat{A} \rightarrow A$ is an isomorphism outside $C$ and that each point of $x \in C$ is replaced by $\mathbb{P}\left(T_{x} / T_{x} C\right)$. Observe further that each entire curve $\gamma: \mathbb{C} \rightarrow A$ lifts to $\widehat{A}$ unless $\gamma(\mathbb{C}) \subset C$. Combined, these facts yield statement 1 ).

2) We have $\pi^{-1}(x) \simeq \mathbb{P}_{1}$ for every $x \in C$. This implies that there is a Brody curve through every point in $E=\pi^{-1}(C)$. Conversely, let $\widehat{f}: \mathbb{C} \rightarrow \widehat{A}$ be a Brody curve. We will see that $\widehat{f}(\mathbb{C}) \subset E=\pi^{-1}(C)$ if we choose $A$ and $C$ according to proposition 7 below. Now $\widehat{f}$ being a Brody curve implies that $f=\pi \circ \widehat{f}: \mathbb{C} \rightarrow A$ is a Brody curve or constant. Let us assume that $f$ is not constant. If $A=\mathbb{C}^{3} / \Gamma$, then $f$ lifts to an affine-linear map $F: \mathbb{C} \rightarrow \mathbb{C}^{3}$. The image $f(\mathbb{C})$ is thus the orbit of a complex one-parameter subgroup $P$ of $A$. Let $H$ denote the (real) closure of $P$ in $A$. Thanks to proposition 7 we may assume that $H$ and $C$ intersect transversally in some point $p$. But now we arrive at a contradiction because according to proposition 3 under these circumstances $f: \mathbb{C} \rightarrow A$ can not be induced by a Brody curve $\widehat{f}: \mathbb{C} \rightarrow \widehat{A}$. Thus $f=\pi \circ \widehat{f}$ must be constant. Since $\widehat{f}$ is non-constant and $\pi$ is an isomorphism outside of $C$, it follows that $\widehat{f}(\mathbb{C}) \subset E=\pi^{-1}(C)$.

5.2. Local model of blow-up. - The idea we use is: If we blow up something, the hermitian metric will explode somewhere. We will now make this precise.

Proposition 2. - Let $A$ be a three-dimension complex manifold, $C$ a smooth curve, $\pi: \widehat{A} \rightarrow A$ the corresponding blow-up with center $C, p \in C$ and $L_{n} a$ sequence of (local) curves converging to a (local) curve $L_{0}$ such that

1) $L_{0}$ intersects $C$ transversally in $p$,

2) the intersection $L_{n} \cap C$ is empty for all $n \neq 0$.

Furthermore assume $A$ and $\widehat{A}$ endowed with hermitian metrics. Then there exist sequences $p_{n} \in L_{n}$ and $v_{n} \in T_{p_{n}}\left(L_{n}\right)$ such that $\lim p_{n}=p$ and 


$$
\limsup \frac{\left\|\pi^{-1}\left(v_{n}\right)\right\|_{\widehat{A}}}{\left\|v_{n}\right\|_{A}}=\infty
$$

(note that $L_{n} \subset A \backslash C$ and that $\pi$ is an isomorphism on $A \backslash C$.)

Proof. - Let $\widehat{p}$ denote the point in $\pi^{-1}(p)$ which points in the direction of $L$ (using the isomorphism between $\pi^{-1}(p)$ and the projectivization of the normal tangent space $T_{p} A / T_{p} C$.)

We fix local holomorphic coordinates on $A$ and $\widehat{A}$ around $p$ resp. $\widehat{p}$ such that the defining equations for $C$ and $L_{0}$ become as simply as possible. Doing this we get local holomorphic coordinates such that

$$
C=\left\{\left(z_{1}, z_{2}, z_{3}\right): z_{1}=z_{2}=0\right\}, \quad L_{0}=\left\{\left(z_{1}, z_{2}, z_{3}\right): z_{2}=z_{3}=0\right\} .
$$

Now the projection map can be written as

$$
\pi\left(x_{1}, x_{2}, x_{3}\right)=\left(x_{1} x_{2}, x_{2}, x_{3}\right) .
$$

Since $\lim L_{n}=L_{0}$, the curves $L_{n}$ can be parametrized as

$$
L_{n}=\left\{\gamma_{n}(t)=\left(t, \alpha_{n}(t), \beta_{n}(t)\right\}\right.
$$

where $t$ runs through an appropriate small neighbourhood of 0 and where $\alpha_{n}$, $\beta_{n}$ are sequences of holomorphic functions converging uniformly to the constant function zero on this small neighbourhood.

Since all the calculations happen in some small neighbourhood of $p$ (resp. $\widehat{p})$, we may replace the given hermitian metrics by the euclidean metric with respect to our coordinate systems.

Our next step is to define the auxiliary function

$$
\phi_{n}(t)=t+\alpha_{n}(t) \alpha_{n}^{\prime}(t)
$$

We observe that $\phi_{n}$ converges to the identity map $\phi(t)=t$. Therefore the theorem of Rouché allows us to choose a sequence $s_{n}$ with $\lim _{n} s_{n}=0$ and $\phi_{n}\left(s_{n}\right)=0$ for all $n$.

We claim: $\alpha_{n}\left(s_{n}\right) \neq 0$. Indeed, assume $\alpha_{n}\left(s_{n}\right)=0$. Then

$$
\alpha_{n}\left(s_{n}\right) \alpha_{n}^{\prime}\left(s_{n}\right)=0
$$

and consequently $0=\phi_{n}\left(s_{n}\right)=s_{n}+0$ implies $s_{n}=0$ and therefore

$$
0=\alpha_{n}\left(s_{n}\right)=\alpha_{n}(0) \text {. }
$$

But $\alpha_{n}(0)=0$ is impossible, because $L_{n} \cap C$ is empty. Thus the assumption $\alpha_{n}\left(s_{n}\right)=0$ leads to a contradiction, i.e., $\alpha_{n}\left(s_{n}\right)$ must be non-zero.

Hence we may divide by $\alpha_{n}\left(s_{n}\right)$ and thereby deduce that $\phi_{n}\left(s_{n}\right)=0$ implies

$$
\alpha_{n}^{\prime}\left(s_{n}\right)=-s_{n} / \alpha_{n}\left(s_{n}\right) \text {. }
$$

TOME $135-2007-\mathrm{N}^{\circ} 1$ 
If $\widehat{\gamma}(t)$ denotes the point in $\widehat{A}$ lying above $\gamma_{n}(t) \in A \backslash C$, we obtain

$$
\widehat{\gamma}_{n}\left(s_{n}\right)=\left(s_{n} / \alpha_{n}\left(s_{n}\right), \alpha_{n}\left(s_{n}\right), \beta_{n}\left(s_{n}\right)\right)=\left(-\alpha_{n}^{\prime}\left(s_{n}\right), \alpha_{n}\left(s_{n}\right), \beta_{n}\left(s_{n}\right)\right)
$$

which converges to $(0,0,0)=\widehat{p} \in \widehat{A}$ if $n$ goes to infinity.

Now

$$
\gamma_{n}^{\prime}\left(s_{n}\right)=\left(1, \alpha_{n}^{\prime}\left(s_{n}\right), \beta_{n}^{\prime}\left(s_{n}\right)\right) \Longrightarrow \lim _{n}\left\|\gamma_{n}^{\prime}\left(s_{n}\right)\right\|=1
$$

while

$$
\widehat{\gamma}_{n}^{\prime}\left(s_{n}\right)=\left(\frac{1-\alpha_{n}^{\prime}\left(s_{n}\right)}{\left(\alpha_{n}\left(s_{n}\right)\right)^{2}}, \alpha_{n}^{\prime}\left(s_{n}\right), \beta_{n}^{\prime}\left(s_{n}\right)\right) \Longrightarrow \lim _{n}\left\|\widehat{\gamma}_{n}^{\prime}\left(s_{n}\right)\right\|=+\infty .
$$

\subsection{Brody curves and blow ups}

Proposition 3. - Let $A$ be an abelian variety, $C$ a submanifold containing $e_{A}$ and $\pi: \widehat{A} \rightarrow A$ the blow-up with center $C$. Let $\phi: \mathbb{C} \rightarrow A$ be a complex 1-parameter subgroup with closure $B=\overline{\phi(\mathbb{C})}$. Let $q \in B$ and define a Brody curve $\gamma: \mathbb{C} \rightarrow A$ by $\gamma(z)=\phi(z)+q$. Assume moreover that

1) $v=\phi^{\prime}(0) \notin T_{e_{A}} C$ and

2) $T_{e_{A}} B \not \subset T_{e_{A}} C \oplus\langle v\rangle_{\mathbb{C}}$.

Then there does not exist a Brody curve $\widehat{\gamma}: \mathbb{C} \rightarrow \widehat{A}$ with $\gamma=\pi \circ \widehat{\gamma}$.

Proof. - Let $\tau: \mathbb{C}^{3} \rightarrow A$ be the universal covering. Recall that $B$ is a real subtorus. Let $V$ be the Lie algebra of $B$. We may regard $V$ as the connected component of $\tau^{-1}(B)$ which contains 0 . Since $\phi(\mathbb{C})$ is dense in $B$ and $q \in B$, we can find elements $\lambda_{n} \in \phi(\mathbb{C})$ such that $\lim \left(\lambda_{n}+q\right)=e_{A}$. Due to condition 2) the elements $\lambda_{n}$ can be chosen in such a way that there are small open neighbourhoods $W$ of $e_{A}$ in $A$ and $\Delta$ of 0 in $\mathbb{C}$ such that

$$
C \cap W \cap\left(\lambda_{n}+\gamma(\Delta)\right)=\{\} .
$$

Now we can use proposition 2 with $p=e_{A}, L_{0}=\phi(\Delta)$ and $L_{n}=\left(\lambda_{n}+\gamma(\Delta)\right)$. Hence

$$
\sup _{t \in \mathbb{C}} \frac{\left\|\widehat{\gamma}^{\prime}(t)\right\|_{\widehat{A}}}{\left\|\gamma^{\prime}(t)\right\|_{A}}=+\infty
$$

where $\widehat{\gamma}: \mathbb{C} \rightarrow \widehat{A}$ is the natural lift of $\gamma$.

Since $\gamma$ is induced by an affine-linear map, the norm $\left\|\gamma^{\prime}(t)\right\|_{A}$ is a positive constant and in particular bounded from below by a number greater than zero. Together with the above equation this implies that $\widehat{\gamma}$ can not be a Brody curve. 
5.4. Excluding real subtori of dimension 3. - In this section we deduce the following statement:

Proposition 4. - There exists an abelian 3-fold $A$ such that every real subtorus of real dimension 3 is totally real in $A$.

We will prove this assertion by showing that every very general abelian three-fold has this property, i.e., we demonstrate:

Proposition 5. - Let $U \rightarrow D$ be a locally complete family of abelian varieties of dimension 3. Then there exists a countable family of nowhere dense closed analytic subsets $Z_{i} \subset D$ such that every abelian threefold $A$ corresponding to a point outside the union $\bigcup_{i} Z_{i}$ has the property "Every real subtorus of real dimension 3 is totally real in $A$ ".

Before proving the proposition, we need some lemmata.

Lemma 2. - Let $A=\mathbb{C}^{3} / \Gamma$ be an complex abelian 3-fold, $S$ a real subtorus of dimension 3. Then there is a joint deformation of $S \subset A$ over the unit disc such that $A_{t}$ is an abelian variety for all $t$ and $S_{t}$ is totally real for all $t \neq 0$.

Proof. - Let $\Lambda \subset \Gamma$ be the $\mathbb{Z}$-submodule corresponding to $S$. Since $A$ is an abelian variety, $\mathbb{C}^{3}$ admits a hermitian form $H$ such that $B=\operatorname{Im} H$ has integer values on $\Gamma \times \Gamma$. Now $B$ is alternating and $3=\operatorname{rank}_{\mathbb{Z}}(\Lambda)$ is odd, hence there is an element $v \in \Lambda$ for which $B(v,$.$) vanishes identically on \Lambda$. Let $\Lambda_{\mathbb{R}}\left(\operatorname{resp} . \Lambda_{\mathbb{C}}\right)$ be the real (resp. complex) vector subspace of $\mathbb{C}^{3}$ generated by $\Lambda$. We may assume that $\Lambda_{\mathbb{R}}$ is not totally real. Then $\operatorname{dim}_{\mathbb{C}}\left(\Lambda_{\mathbb{C}}\right)=2$ and $L=\Lambda_{\mathbb{R}} \cap i \Lambda_{\mathbb{R}}$ is a complex line. Now we choose an element $w \in \Gamma$ such that

1) $B(v, w) \neq 0$,

2) $B(w,$.$) does not vanish identically on L$ and

3) $w \notin \Lambda_{\mathbb{C}}$.

We define $\mathbb{R}$-linear self-maps $\phi_{t}$ of $\mathbb{C}^{3}$ as follows: First we observe that $\mathbb{C}^{3}$ is the direct sum of $\mathbb{R} \cdot v$ and $K=\{x: B(x, w)=0\}$. Second we set

$$
\phi_{t}(v)=v+t w \quad \text { and } \quad \phi_{t}(x)=x \text { for all } x \in K .
$$

It is easy to check that $\phi_{t}$ is always bijective and moreover an isometry for $B$. Hence $\Gamma_{t}=\phi_{t}(\Gamma)$ is a lattice for which the assertion $B\left(\Gamma_{t}, \Gamma_{t}\right) \subset \mathbb{Z}$ holds. Thus $A_{t}=\mathbb{C}^{3} / \Gamma_{t}$ is an abelian variety.

Now let us look at $\phi_{t}\left(\Lambda_{\mathbb{R}}\right)$. First we consider the real vector subspace

$$
V=\Lambda_{\mathbb{R}} \oplus \mathbb{R} w .
$$

Let $K=\{x: B(x, w)=0\}$. Then $V=(V \cap K) \oplus \mathbb{R} v$. Now $\phi_{t}$ acts trivially on $K$ and $\phi_{t}(v)=v+t w \in V$. Hence $\phi_{t}$ stabilizes $V$. We note that $\operatorname{dim}_{\mathbb{R}}(V)=4$ and $\langle V\rangle_{\mathbb{C}}=\mathbb{C}^{3}$, because $w \notin \Lambda_{\mathbb{C}}$. Therefore $V$ contains a unique complex 
line, which must be $L$. Since $\phi_{t}\left(\Lambda_{\mathbb{R}}\right) \subset V$, we may deduce that for each $t$ either $\phi_{t}\left(\Lambda_{\mathbb{R}}\right)$ is totally real or contains $L$. Now, by the construction of $\phi_{t}$ it is clear that

$$
\phi_{t}\left(\Lambda_{\mathbb{R}}\right) \cap \phi_{s}\left(\Lambda_{\mathbb{R}}\right)=\Lambda_{R} \cap K
$$

for any $s \neq t$. Since $L \neq \Lambda_{\mathbb{R}} \cap K$ due to condition 2) for the choice of $w$, we may deduce that $L \not \subset \phi_{t}\left(\Lambda_{R}\right)$ for $t \neq 0$. As a consequence, $\phi_{t}\left(\Lambda_{\mathbb{R}}\right)$ is totally real for $t \neq 0$.

Lemma 3. - Let $\pi: U \rightarrow D$ be a family of 3-dimensional complex abelian varieties, parametrized by $D$ which we assume to be the unit ball in some $\mathbb{C}^{N}$. Let $S_{0}$ be a real 3-dimensional subtorus of the abelian variety $U_{0}=\pi^{-1}(0)$ Then there is natural deformation $S_{t}$ of $S_{0}(t \in D)$ such that

$$
Z=\left\{t \in D: S_{t} \text { is not totally real }\right\}
$$

is a closed complex analytic subset of $D$.

Proof. - The family $U$ can be described as a quotient $\mathbb{C}^{3} \times D$ by a $\mathbb{Z}^{6}$-action which is given as

$$
\left(m_{1}, \ldots, m_{3}, n_{1}, \ldots, n_{3}\right):(v ; t) \longmapsto\left(v+\left(m_{1}, m_{2}, m_{3}\right)+\sum_{i} n_{i} f_{i}(t) ; t\right)
$$

where $v \in \mathbb{C}^{3}, t \in D$ and where the $f_{i}$ are holomorphic maps from $D$ to $\mathbb{C}^{3}$.

We may assume that $S_{0}$ is the subtorus for which the corresponding subgroup of $\mathbb{Z}$-rank 3 is generated by $f_{1}(0), f_{2}\left(0\right.$ and $f_{3}(0)$. Then $S_{t}$ corresponds to the subgroup generated by the $f_{i}(t)$ and $S_{t}$ is totally real if and only if this group spans $\mathbb{C}^{3}$ as a complex vector space. Therefore the set of all $t \in D$ for which $S_{t}$ fails to be totally real is the zero locus of $\left.\operatorname{det}\left(f_{1}(t), f_{(} t\right), f_{3}(t)\right)$ and thus is a closed complex analytic set.

Now we can prove the proposition.

Proof. - There are only countably many different real subtori of real dimension three for a given abelian 3 -fold $A$, each corresponding to a $\mathbb{Z}$-submodule of rank three of $\mathbb{Z}^{6}=H_{1}(A, \mathbb{Z})$.

Inside the family $U \rightarrow D$ there are canonical isomorphisms

$$
H^{1}\left(U_{0}, \mathbb{Z}\right) \simeq H^{1}\left(U_{t}, \mathbb{Z}\right)
$$

which we may therefore identify.

Now for each fixed $\mathbb{Z}$-submodule of rank three the set of all $t \in D$ for which the corresponding subtorus $S_{t}$ fails to be totally real is a closed analytic subset (lemma 3 ) which is not all of $D$ (lemma 2). This proves the proposition 5 and thereby proposition 4 . 
REMARK. - 1) Since every subtorus of dimension smaller than 3 can be embedded into a subtorus of dimension 3, the property "All real subtori of real dimension 3 are totally real" is equivalent to the property "All real subtori of real dimension up to 3 are totally real".

2) An abelian threefold $A$ is a simple abelian variety if and only if it contains no elliptic curve. The latter property is equivalent to the statement "All real subtori of real dimension up to two are totally real". Hence the property "All real subtori of real dimension three are totally real" implies that the abelian 3 -fold under discussion is simple.

5.5. Dealing with real subtori of dimension 4. - The main goal of this section is to to verify that we can a choose a curve $C$ in a 3 -dimensional abelian variety $A$ such that $C$ intersects the closure of every translate of every real subtorus of real dimension 4 .

Lemma 4. - Let $A$ be an abelian threefold, $x \in A$ and let $L$ be a complex line in $T_{x} A$. Then there exist smooth curves $C \subset A$ with $x \in C$ such that $T_{x} C$ is arbitrarily close to $L$.

Proof. - We construct curves by embedding $A$ into a projective space and taking the intersection of $A$ with linear subspaces of codimension two containing $x$. Then the statement follows from Bertini's theorem.

Lemma 5. - Let $A$ be an abelian threefold with smooth curves $C$ and $C^{\prime}$. Then there is a dense open subset $U \subset A$ such that $C \cup \lambda_{t}^{*} C^{\prime}$ is smooth for $t \in U$ where $\lambda_{t}$ denotes translation by $t$.

Proof. $-C \cup \lambda_{t}^{*} C^{\prime}$ is smooth if and only if $C$ and $\lambda_{t}^{*} C^{\prime}$ are disjoint. Hence $U=A \backslash\left\{x-y: x \in C, y \in C^{\prime}\right\}$.

LEMma 6. - Let $V$ be a complex 3-dimensional vector space equipped with a hermitian inner product $H$ and let $W$ be a real 4-dimensional real subspace. Then there exists a complex line $L \subset V$ such that the angle between $W$ and $L$ is at least $\frac{1}{4} \pi$. i.e.,

$$
|\langle v, w\rangle| \leq \cos \left(\frac{1}{4} \pi\right)\|v\| \cdot\|w\|
$$

for all $v \in L, w \in W$.

REMARK. - If $H(.,$.$) is an hermitian inner product, its real part is the asso-$ ciated euclidean inner product and thus the angle between two vectors $v$ and $w$ is the number $\phi \in\left[0, \frac{1}{2} \pi\right]$ for which $\cos \phi=\operatorname{Re} H(v, w)$.

Proof. - We may choose vectors $A, B, C$ such that $(A, i A, B, i B, C, i C)$ is an orthonormal basis for $\operatorname{Re} H$ and $\langle A, i A, B, C+\lambda i B\rangle=W$ for some $\lambda \in \mathbb{R}$. Then we choose: 
- $L=\langle C\rangle_{\mathbb{C}}$ if $|\lambda|>1$,

- $L=\langle B+i C\rangle_{\mathbb{C}}$ if $0 \leq \lambda \leq 1$ and

- $L=\langle B-i C\rangle_{\mathbb{C}}$ if $-1 \leq \lambda<0$.

It is easy to check that in each case the angle is at least $\frac{1}{4} \pi$.

Proposition 6. - Let $A$ be an abelian threefold (i.e. an abelian variety of dimension 3). Then there exists a smooth complex curve $C \subset A$ such that for every real 4-dimensional subtorus $S \subset A$ and every point $a \in A$ there exists a point $p \in C$ where $C$ and $S(a)$ (the $S$-orbit in $A$ through a) intersect transversally.

Proof. - We have to consider all 4-subtori. Since the set of all such tori lacks good geometric properties, we instead consider the larger set of all connected real Lie subgroups of real dimension 4, or, equivalently, the real Grassmann variety $M$ which parametrizes all real vector subspaces of dimension 4 of the Lie algebra $\operatorname{Lie}(A) \simeq \mathbb{C}^{3}$. This is a real compact variety.

Now we fix an hermitian inner product on $\operatorname{Lie}(A) \simeq \mathbb{C}^{3}$ (e.g. the standard one for $\mathbb{C}^{3}$ or the one corresponding to the Riemann condition). For each element $H \in M$ we define a closed neighbourhood $B_{H}$ as follows: An element $H^{\prime}$ belongs to $B_{H}$ iff for every vector $v^{\prime}$ in $H^{\prime}$ there is a vector $v \in H$ such that the angle between $v$ and $v^{\prime}$ is at most $\frac{1}{16} \pi$. Due to compactness of $M$ there is a finite collection of elements $H_{i} \in M, i \in I$ such that $M=\bigcup_{i \in I} B_{H_{i}}$.

Next we will choose a smooth complex curve $C_{i} \subset A$ for each $i \in I$. Fix an index $i$. Let $H=H_{i}$ and $B=B_{H_{i}}$. Choose a complex line $L$ in $T_{e_{A}} A \simeq \operatorname{Lie}(A)$ such that the angle between $L$ and $H$ is at least $\frac{1}{4} \pi$ (which is possible due to lemma 6). Then we choose a smooth complex curve $S=S_{i}$ through $e_{A}$ such that for each $v \in T_{e_{A}} S$ there is a vector $v^{\prime} \in L$ such that the angle between $v$ and $v^{\prime}$ is at most $\frac{1}{16} \pi$ (lemma 4). By the definition of $B$, the angle between $T_{e_{A}} S$ and $H^{\prime}$ is at least $\frac{1}{8} \pi$ for every $H^{\prime} \in B$.

Now let $\pi: \mathbb{C}^{3} \rightarrow A$ denote the universal covering. Let $F$ denote a fundamental region, i.e. a compact subset of $\mathbb{C}^{3}$ with $\pi(F)=A$. Let $W$ be an open neighbourhood of $e_{A}$ in $S$ which is small enough such that the embedding of $W$ in $A$ lifts to an embedding into $\mathbb{C}^{3}$, taking $e_{A}$ to 0 . In addition, we require that $W$ is small enough such that for every $w \in W, v \in T_{w} S \backslash\{0\}$ and $v^{\prime} \in T_{e_{A}} S \backslash\{0\}$ the angle between $v$ and $v^{\prime}$ is at most $\frac{1}{16} \pi$.

For each $H^{\prime} \in B$ we define

$$
Z\left(H^{\prime}\right)=\left\{c+h: c \in W, h \in H^{\prime}\right\} .
$$

We claim: There exists a number $\rho>0$ such that $Z\left(H^{\prime}\right)$ contains the ball with radius $\rho$ and center 0 for every $H^{\prime} \in B$. Indeed, assume the contrary. Then there are sequences $v^{(k)} \in \mathbb{C}^{3}$ and $H^{(k)} \in B$ such that: 
1) $H^{(k)}$ converges to an element $H^{\prime \prime} \in B$ (recall that $B$ is compact),

2) $\lim v^{(k)}=0$,

3) $v^{(k)} \notin Z\left(H^{(k)}\right)$.

But this would contradict the fact that $H^{\prime \prime}$ and $T_{e_{A}} W$ are transversal. Thus we can find such a number $\rho$. Next, using compactness of $F$, we choose a finite set $\Sigma \subset \mathbb{C}^{3}$ such that for every $x \in F$ there is an element $s \in \Sigma$ with $\|x-s\|<\frac{1}{3} \rho$.

Using this fact and lemma 5 we can find a map $\xi: \Sigma \rightarrow \mathbb{C}^{3}$ such that:

- $\bigcup_{s \in \Sigma}(\pi(\xi(s))+S)$ is smooth and

- $|\xi(s)|<\frac{1}{3} \rho$ for all $s \in \Sigma$

Then we have constructed a smooth curve in $A$, namely

$$
S^{\prime}=\bigcup_{s \in \Sigma}(\pi(\xi(s))+S)
$$

with the following property:

(T) For every vector $u \in \mathbb{C}^{3}$ with $\|u\|<\frac{1}{3} \rho$ and every real 4-dimensional subtorus $H$ of $A$ with $\operatorname{Lie}(H) \in B$ every $H$-orbit in $A$ intersects $\pi(u)+S^{\prime}$ in some point transversally.

We found this curve $S^{\prime}$ after fixing an element $i \in I$. We can do the same for every element $i \in I$, obtaining a family of curves $S_{i}^{\prime}$ and a family of positive real numbers $\rho_{i}$.

Then by lemma 5 we can choose vectors $u_{i}$ such that $\left\|u_{i}\right\|<\frac{1}{3} \rho_{i}$ and such that $C=\bigcup_{i \in I}\left(\pi\left(u_{i}\right)+S_{i}^{\prime}\right)$ is a smooth curve.

By construction this curve has the property that it intersects each translate of each real 4-dimensional subtorus of $A$ in at least one point transversally.

Proposition 7. - There exists an abelian threefold $A$ with a complex curve $C$ such that the following property holds: For every complex one-parameter subgroup $P$ of $A$ and every point a in $A$ there is a point $p$ in $C$ where $C$ and the (real) closure of $P \cdot a$ intersect transversally.

Proof. - We may choose $A$ such that every real 3-dimensional real subtorus is totally real (proposition 4). Then evidently real subtori of smaller dimension are totally real as well. Now let $P$ be a complex 1-parameter subgroup of $A$. The closure of $P$ is again a subgroup, and therefore in fact a real subtorus. This subtorus does not need to be complex, but it can not be totally real, since it contains $P$. Therefore for every complex 1-parameter subgroup $P$ of $A$ the real dimension of its closure in $A$ is at least 4 . In particular, the closure $\bar{P}$ of $P$ in $A$ contains a real subtorus $S$ of real dimension 4 . Now it suffices to choose the curve $C$ according to proposition 6: If $\bar{P}$ contains a real subtorus $S$ of real dimension 4 which intersects $C$ transversally in some point $p$, then $\bar{P}$ itself has transversal intersection with $C$ in $p$ as well. 


\section{Brody curves and sets of rational points}

Conjecturally entire curves or Brody curves with values in projective varieties defined over some number field behave somewhat analoguously to sets of rational points (admitting finite field extensions).

As we have seen, Brody curves and arbitrary entire curves behave differently. So which are the right analogue for rational point sets? In our construction at one point we made a "very generic" choice. For this reason it is not clear whether one can find such an example which is defined over a number field.

If such an example can be defined over a number field, it would suggest that complex-analytic concept corresponding to infinite rational point sets are arbitrary entire curves and not Brody curves: For every abelian variety $A$ defined over a number field $k$ there is a finite field extension $K / k$ such that $A(K)$ is Zariski dense. Then also $X(K)$ is Zariski dense in $X$ for every projective manifold $X$ obtained from $A$ by blowing up something. Thus if our construction can be realized over a number field, it would yield a projective variety defined over some number field $K$ such that every Brody curve is degenerate, but there is a Zariski dense subset of $K$-rational points.

In any case, dense sets of rational points as well as dense entire curves behave nicely under birational transformations while our example shows that the behaviour of Brody curves may change dramatically.

This suggests that the right complex-analytic analogue to infinite sets of rational points should be arbitrary entire curves rather than Brody curves.

Acknowledgement. - The author wants to thank V. Bangert and B. Siebert for the invitation to the workshop in Freiburg in September 2003 and to thank Serge Cantat and Frederic Campana for useful discussions and the referee for useful suggestions. The research for this article was done by the author while working at the Institut Élie Cartan.

\section{BIBLIOGRAPHY}

[1] N. U. ARAKELJAN - "Approximation complexe et propriétés des fonctions analytiques", in Actes du Congrès International des Mathématiciens (Nice, 1970), Tome 2, vol. 2, Gauthier-Villars, 1971, p. 595-600.

[2] A. BLOCH - "Sur les systèmes de fonctions uniformes satisfaisant à l'equation d'une variété algébrique dont l'irrégularité dépasse la dimension", J. Math. Pures Appl. 5 (1926), p. 19-66.

[3] R. Brody - "Compact manifolds in hyperbolicity", Trans. Amer. Math. Soc. 235 (1978), p. 213-219. 
[4] F. Campana - "Orbifolds, special varieties and classification theory", Ann. Inst. Fourier (Grenoble) $\mathbf{5 4}$ (2004), p. 499-630.

[5] G. Faltings - "Endlichkeitssätze für abelsche Varietäten über Zahlkörpern", Invent. Math. 73 (1983), p. 349-366.

[6] Y. KawAmata - "On Bloch's conjecture", Invent. Math. 57 (1980), p. 97100.

[7] S. KobAYASHI - Hyperbolic complex spaces, Grundlehren der Mathematischen Wissenschaften, vol. 318, Springer, 1998.

[8] S. LANG - Introduction to complex hyperbolic spaces, Springer, 1987.

[9] _ Number theory. III, Encyclopaedia of Mathematical Sciences, vol. 60, Springer, 1991, Diophantine geometry.

[10] H. L. Royden - "Remarks on the Kobayashi metric", in Several complex variables, II (Proc. Internat. Conf., Univ. Maryland, College Park, Md., 1970), Lecture Notes in Math., vol. 185, Springer, 1971, p. 125-137. Lecture Notes in Math., Vol. 185.

[11] P. VoJTA - Diophantine approximations and value distribution theory, Lecture Notes in Mathematics, vol. 1239, Springer, 1987.

[12] L. Zalcman - "Normal families: new perspectives", Bull. Amer. Math. Soc. (N.S.) 35 (1998), p. 215-230. 